Submitted to: Biosensors \& Bioelectronics

Date: September $27^{\text {th }}, 2004$

Revised form: November, $15^{\text {th }} 2004$

\title{
DNA nanofilm thickness measurement on microarray in air and in liquid using an atomic force microscope
}

GUILLAUME LEGAY $^{(1)}$, ERIC FINOT ${ }^{(1)} *$, RITA MEUNIER-PREST ${ }^{(2)}$, MUSTAPHA $^{(1)}$ CHERKAOUI-MALKI $^{(3)}$, NORBERT LATRUFFE ${ }^{(3)}$ AND ALAIN DEREUX ${ }^{(1)}$.

(1) Laboratoire de Physique, UMR CNRS 5027, Université de Bourgogne, BP 47870, 21078 Dijon, France

(2) Laboratoire de Synthèse et d'Electrosynthèse Organométalliques, UMR CNRS 5188, F21000 Dijon, France

${ }^{(3)}$ Laboratoire de Biologie Moléculaire et Cellulaire, GDR CNRS 2583, F-21000 Dijon, France.

*Corresponding author: Laboratoire de Physique de 1'Université de Bourgogne, UFR Sciences et Techniques, 9 rue A. Savary, BP 47870, 21078 DIJON, France.

E-mail address: Eric.Finot@u-bourgogne.fr 


\section{ABSTRACT}

The measurement of the thickness of DNA films on microarray as a function of the medium (liquid, air) is gaining importance for understanding the signal response of biosensors. Thiol group has been used to attach DNA strands to gold micropads deposited on silicon surface. Atomic Force Microscopy (AFM) was employed in its height mode to measure the change in the pad thickness and in its force mode to measure the indentation depth of the nanofilm. A good coherence between the height and force modes is observed for the film thickness in air. The adhesion force was found to be an alternative way to measure the surface coverage of the biolayer at nanoscopic scale. However the force analysis (compression, steric and electrostatic) provides baseline information necessary to interpret the AFM height image in liquid. Analysis of the film thickness distribution shows that the height of the DNA strands depends on both the DNA strand length (15 to 35 base pairs) and the environment (air, liquid). In air, longer strands lay down onto gold surface whereas the charge reversal of gold in liquid causes a repulsion of longer strands which stand up.

KEY WORDS: DNA microarray; Scanning Force Microscopy; immobilization; gold-thiolate bond.

PACS : 87.15.By; 87.64.D; 83.85 Gk 


\section{Introduction}

Single-stranded DNA (ssDNA) microarrays have been extensively used as chip-scale platforms for interrogation of gene expression, polymorphism analysis, and mutation screening. The DNA probe is immobilized onto a solid support allowing high-density spotting (Lenigk et al., 2001). The automation of array fabrication, data acquisition and analysis becomes therefore evident

Among label free methods that do not use fluorescence (Yoshino et al., 2003), one can discern optical techniques such as spectral ellipsometry (Gray et al., 1997) and/or surface plasmon resonance (Georgiadis et al., 2000; Lotierzo et al., 2004; Bassil et al., 2003) ; mechanical approach as the cantilever deflection (Rasmussen et al., 2003; Cherian et al., 2003 ; Marie et al., 2002) ; or electrochemical response (Meunier-Prest et al., 2003; Liang et al., 2004; Finot et al., 2003; Carpini et al., 2004)

Although all those methods provide average quantitative information, the sensor response cannot be interpreted simply using a single parameter as the mass uptake. Optical sensors are sensitive in their principles to the change in the optical index and thickness of the biolayer immobilized at the sensor surface. Microcantilevers responses are based on the changes in surface stress of the biolayer. Besides strands movement is useful for generating nanomechanical cantilever motion.

The knowledge of the DNA film thickness is therefore of fundamental importance to interpret the changes of the signal issued from label free biosensors. In addition, high density ssDNA coverage may impede the hybridization due to steric effects, which may reduce the effectiveness of the chip. Thus, the control of the surface density as well as the strands orientation towards the surface is highly relevant to avoid that only a part of the immobilized 
ssDNA is able to hybridize to its complementary DNA strand. Therefore, a novel approach is again required to appreciate the orientation of the strand.

A well-known technique for a stable and reproducible DNA immobilization is the chemisorption of thiol modified DNA strands onto a gold surface. The use of gold as a solid support has several advantages compared to silicon wafers (Ulmann et al., 2000; Ulmann, 1991): it is of special interest for surface plasmon resonance, microcantilever fabrication as well as electrochemical measurements.

Surface coverage and the properties of surface immobilized DNA have been studied by a range of techniques that include surface forces (Cho et al., 2001), neutron reflection (Levicky et al., 1998).

Force spectroscopy (Schotanus et al., 2002) has been a useful technique to measure molecular interactions between the probe tip and immobilized biomolecules. The forces between complementary strands have investigated in electrolyte solution (Wang et al., 2001). The height of long DNA molecules adsorbed on mica was investigated in AFM (MorenoHerrero et al., 2003).

Although the self-assembly of thiol group on gold surface is well established and has been investigated previously by AFM (Satjapipat et al., 2001; Shi et al., 2002; Zhou et al, 2002), little is known about the layer thickness as a function of the sequence length and the data concerning the immobilized ssDNA are sparse (Holmberg et al., 2003; O'Brien et al., 2000).

Our strategy combines the ability of the AFM to detect changes both in topography and in surface forces with the ease to patterning gold clusters on surface. 
Herein we report on a novel approach for measuring the thickness distribution of an oligonucleotide film attached onto a gold substrate. The adhesion force in air and the repulsive force in liquid have been studied as a function of the ssDNA sequence length.

\section{Experimental}

\subsection{Array fabrication}

Electron beam (e-beam) lithography has been used to fabricate $4 \times 4$ array of gold squared pads that are $5 \mu \mathrm{m}$ in width and with a $20 \mu \mathrm{m}$ center-to-center spacing.

First, a 300-nm thick layer of $50 \mathrm{kD}$ molecular weight of PolyMethylMethAcrylate (PMMA) resist (4\% content of polymer in chlorobenzene) was spin coated onto the silicon substrate. The sample was then baked for $3 \mathrm{~h}$ at $175^{\circ} \mathrm{C}$ before being patterned by an electron beam. An electron beam with a diameter of $10 \mathrm{~nm}$ or less is used to irradiate, and thereby chemically modify the PMMA layer. A conventional Raith Elphy plus pattern generator, in connection with a JEOL 840 scanning electron microscope equipped with a thermal field emitter and having a nominal resolution of $5 \mathrm{~nm}$, was used to expose the PMMA layer. The beam energy was $20 \mathrm{keV}$ and the beam current was set at $0.18 \mathrm{nA}$. Two exposure fields were used to match the resolution requirements of the structures. The structures were developed in MIBK for $40 \mathrm{~s}$, rinsed in isopropanol for $30 \mathrm{~s}$ and blown dry with pure nitrogen. $\mathrm{Cr}(10 \mathrm{~nm})$ and $\mathrm{Au}(40 \mathrm{~nm})$ were evaporated in a thermal evaporator (e-gun) and the remaining resist was lifted-off in trichloroethylene. All the substrates were cleaned with hot acetone, rinsed with isopropyl alcohol and dried on a hot plate at $105^{\circ} \mathrm{C}$.

\subsection{DNA grafting}


Freshly evaporated gold electrodes were modified by incubation in $0.1 \mathrm{mM}$ solutions of thiolated ssDNA in $5 \mathrm{mM}$ potassium phosphate buffer / $50 \mathrm{mM} \mathrm{NaCl}(\mathrm{pH} 7.4)$ for 18-48 $\mathrm{h}$ at ambient temperature. The DNA sequence for 15 bases is 5'-AGT ACA GTC ATC GCG-3, for 35 bases : 5' -TTT TTT TTT TTT ATT AAC CCT CAC TAA AGC GCG CA-3' Modified micropads were rinsed in $5 \mathrm{mM}$ potassium phosphate buffer, $\mathrm{pH} 7.4$ prior to use.

\subsection{Sample characterization}

All experiments were carried out in a Nanoscope D3100 AFM (Digital Instruments) equipped with a $100 \mu \mathrm{m}$ scanner. Unsharpened "D" type silicon nitride cantilevers (microlevers with nominal end-radius $\sim 25 \mathrm{~nm}$ and a nominal force constant of $0.032 \mathrm{~N} . \mathrm{m}^{-1}$ estimated). The height scale was calibrated using colloidal gold spheres of well-defined size (diameter 5 and $14 \mathrm{~nm}$ ). The scan rate was fixed to $20 \mu \mathrm{m} / \mathrm{s}$. All imaging and force measurements were performed in air or in a glass fluid cell (Digital Instruments) at $25^{\circ} \mathrm{C}$.

AFM was specifically chosen so that we could interrogate the 3D character of the surface: AFM allows direct measurement of the area, depth and volume of surface features. Thus, the evolution of the surface roughness can be followed in detail, and surface profiles over different lines in the scans can be evaluated.

Statistical data were extracted from a very large set of measurements: for each experiment, data from 20 different $5 \times 5 \mu \mathrm{m}$ areas were typically combined to generate the data for statistical analyses. The height distributions of the surface were evaluated by measuring the height difference along 50 scan lines of $50 \mu \mathrm{m}$ in length and from these data making histograms.

Root-mean-square (RMS) surface roughness, which is typically used to quantify variations in surface elevation, was directly determined from the height data as: 
$R M S=\sqrt{\prime}$

where $Z_{i}$ is the surface elevation, $Z_{\text {arg }}$ is the mean value of $Z_{i}$ and $N$ represents the sample number.

In order to provide a more meaningful description of the surface, it is instructive to examine the complete height histogram of the image. A height histogram of the image can also be used to reconstruct height values and mean height, and thus represents a superset of the data contained in a RMS surface roughness value.

The Gaussian distribution takes the following form:

$$
P(x)=C
$$

where $P(x)$ is the number of samples at the particular point $x$ in the histogram, $\mu$ the mean height value, $\sigma$ the standard deviation and $\mathrm{C}$ the normalization constant.

The atomic force microscope was also used as a force apparatus. Force measurements were performed on DNA samples in air and in aqueous solutions. The experiment consists in monitoring the interaction between the AFM tip and the substrate by sensing the cantilever deflection, $Z_{c}$, as a function of the piezo elongation, $Z_{p}$, as the tip is moved towards and away from the substrate. Data were collected over a time period of 2 hours and a total of 100 forces curves were analysed for each sample in a given environment.

As the tip-sample separation cannot be independently measured, we developed a systematic procedure for calculating the sensitivity of the apparatus. To avoid the subjectivity of the operator, all force-distance curves were automatically analysed using a procedure programmed under Matlab environment. This measurement assumes that the tip and the gold 
surface are brought into a nondeformable contact in the higher loading region. Raw data $\left(Z_{c}\right.$ versus $Z_{p}$ ) are then converted into force $F$ versus surface-tip separation $D$ using Hooke's law, $F=k Z_{c}$, where $k$ is the spring constant of the cantilever, and the geometric relationship $D=Z_{c^{-}}$ $Z_{p}$ for incremental changes.

\section{Results and discussion}

\subsection{Thickness distribution of the gold plot in air}

The accuracy of the method was tested by determining the thickness distribution of the gold film deposited on silicon substrate. The silicon surface is nearly flat and covered by a thin amorphous layer of $\mathrm{SiO}_{2}$ with a surface roughness of $0.15 \mathrm{~nm}$. The thickness distribution is mainly controlled by the gold morphology. Our goal was to minimize the surface roughness of the gold patterns. The $\mathrm{Au}$ film deposited on Si-(111) presents a columnar structure characterized by fine beads. Grain size and film roughness are mainly explained in terms of surface diffusion, grain aggregation and growth rate of the film that depend on the deposition temperature and the deposition rate. Since the lithography process requires no heating of the polymer mask above $180^{\circ} \mathrm{C}$, the gold layer was deposited at $40^{\circ} \mathrm{C}$ but at the lowest deposition rate possible $(0.1 \mathrm{~nm} / \mathrm{s})$. In this case, the surface diffusion is significant and the deposited species do not have enough time to grow into large grains, so that the grain aggregation is not obvious.

It is important to characterize the surface roughness $\sigma^{2}$ using intrinsic parameters which are independent of the surface area $a$ or the sampling length. In this case, $\log \left(\sigma^{2}\right)$ varies linearly when increasing $\log (a)$ and the slope of the curve indicates a fractal dimension of 0.44 
Figure $1 \mathrm{~A}$ is an AFM image (512x512 pixels) of the $4 \times 4$ array of gold pads that covers $30 \%$ of the observed area $(100 \mu \mathrm{m} \times 100 \mu \mathrm{m})$. The size of the gold grains was found to be identical for both the micropads and the continuous film, which means that the lithography process does not influence the roughness of the deposit. The height analysis issued from Figure 1A is performed over a central area of $4 \mathrm{x} 4 \mu \mathrm{m}^{2}$ for each pad, avoiding the convolution between the AFM probe and the pad boundaries. This step previously requires the location of the pad centers, and a planefit (order 2) of the silicon substrate provides the zero on the height scale. The pad height corresponds to the difference between the silicon fit and the height measured for the pad. Figure 1B shows a typical histogram of the pad thickness. The bin size is determined statistically by dividing the range of measurements by the square-root of the number of measurements. A bin size between 0.1 and $0.2 \mathrm{~nm}$ was chosen as a suitable value to analyze the statistical law of Poisson. The mean value of the thickness is randomly distributed between 54.6 and $54.9 \mathrm{~nm}$ whatever the number of pads analyzed $n$. However, the standard deviation $\sigma$ appears to increase with increasing $n$. The pad area is $(4 \mathrm{x} 4) \mu \mathrm{m}^{2}$. Figure 1C compares the values of $\sigma$ obtained with increasing $n$ with the surface roughness corresponding to continuous film. It indicates that the surface roughness and $\sigma$ have a certain extent relation above 4 pads. Below $n=4$, the number of samples $N$ being less than 75 is not sufficient to represent all data.

A single pad study performed on a $(16 \mu \mathrm{m} \times 16 \mu \mathrm{m})$ image indicates that the height distribution remains identical whatever the sample sizes $(512 \times 512,256 \times 256,128 \times 128)$. However, as it will be shown later, the adsorption of some salt crystals on pad surface during the DNA grafting may perturb the analysis in liquid environment. This is the reason why, in the further studies, we analyse 10 non-contaminated pads on the array. Enlarging the observed surface offers another advantage since a better reproducibility was obtained in the roughness regime which is only slightly related to the surface area. 
No thickness variation was detected as a function of the applied force in the $50 \mathrm{pN}$ to $10 \mathrm{nN}$ range, indicating that the AFM probe does not indent the Au surface. Moreover the thickness of the native gold pad was found to be independent of the scan rate in the 0.1 and 1 $\mathrm{Hz}$ range.

\subsection{Thickness distribution of DNA film in air}

As the thiol-modified DNA strands adsorb onto gold surfaces to form a Self Assembled Monolayer (SAM) but not on silicon, the thickness of the DNA layer is easily obtained from the difference between the thicknesses of the native gold pad and grafted pad. The scan rate and the force applied by the AFM probe were found to be the most critical parameters affecting the height distribution, as expected for a smooth surface.

The applied force $F_{a p p}$ is likely the main factor influencing the measurement of the DNA thickness. It was essential that the set-point which controls $F_{a p p}$ was regularly monitored and adjusted if necessary, and could go over the full image. Note that this procedure is more delicate to maintain in air than in liquid environment. For $F_{a p p}>10 n N$, the tip removes the film in the scanned region through a physical scratching mechanism and deposits are visualized at the boundaries of the image. For $F_{a p p}$ of approximately $1 \mathrm{nN}$, the pad thicknesses do not change in the presence of DNA strands, indicating that the DNA strands are pushed down by the tip in a reversible mechanism since the initial thickness of the DNA strands is recovered with decreasing $F_{a p p} . F_{a p p}=100 \mathrm{pN}$ appears as the optimum setting-point. At the level of low forces, the problem lies in the drift of $F_{a p p}$ during the acquisition time.

The scan rate is a second-order parameter: $\mu$ varies by $0.5 \mathrm{~nm}$ within the $[0.1-2] \mathrm{Hz}$ range. The sweep frequency is chosen at $1 \mathrm{~Hz}$ because the lowest scan rates induce a drift of $F_{a p p}$ over large scan size and the fast scanning conditions do not allow the piezotube to track accurately the topography. 
The height distributions for the 15, 25 and 35 strands, in air, are shown in Figure 2A, whereas the parameters of the fitted Gaussian distribution are collected in Table I.

Table I: Characteristics of the thickness distribution in air and in liquid.

\begin{tabular}{|c|c|c|c|}
\hline \multirow{3}{*}{$\mathrm{Au}$} & & $\mu(\mathrm{nm})$ & $\sigma(\mathrm{nm})$ \\
\hline & air & 54.5 & 1.4 \\
\hline & liquid & 54.5 & 1.5 \\
\hline \multirow[t]{2}{*}{$\mathrm{Au}+(\mathrm{DNA}) 15 \mathrm{ss}$} & air & 57.9 & 2.03 \\
\hline & liquid & 58.0 & 3 \\
\hline \multirow[t]{2}{*}{$\mathrm{Au}+(\mathrm{DNA}) 25 \mathrm{ss}$} & air & 56.7 & 1.87 \\
\hline & liquid & 60 & 2.5 \\
\hline \multirow[t]{2}{*}{$\mathrm{Au}+(\mathrm{DNA}) 35 \mathrm{ss}$} & air & 56.0 & 1.32 \\
\hline & liquid & 64 & 2 \\
\hline
\end{tabular}

The (DNA) ${ }_{15 \mathrm{ss}}$ modified micropads undergo the biggest change in thickness $(3.5 \mathrm{~nm})$, while the two other films are more or less $2 \mathrm{~nm}$ high. Assuming a distance between bases of $0.34 \mathrm{~nm}$ and a thiol length of $1 \mathrm{~nm}$, the expected length of the strands is reported in Table II.

Table 2: Characteristics of the thickness distribution of the layer in air and in liquid measured in height mode $(\mathrm{H})$ and in force mode $(\mathrm{F}) . \mathrm{L}$ is the expected length of the unfolded single strands.

$\alpha$ is the effective orientation of the strands towards the substrate plane in liquid.

\begin{tabular}{|l|l|l|c|c|}
\hline & $\begin{array}{l}\text { AIR } \\
(\mathrm{nm})\end{array}$ & $\begin{array}{l}\text { LIQUID } \\
(\mathrm{nm})\end{array}$ & $\begin{array}{c}\mathrm{L} \\
(\mathrm{nm})\end{array}$ & $\begin{array}{c}\alpha \\
\left({ }^{\circ}\right)\end{array}$ \\
\hline \multirow{2}{*}{ (DNA)15ss } & $\mathrm{H}: \quad 3.4 \pm 1.3$ & $3.4 \pm 3$ & 5.8 & $35 \pm 30$ \\
\cline { 2 - 3 } & $\mathrm{F}: 4.5$ & 4.5 & & \\
\hline \multirow{2}{*}{ (DNA)25ss } & $\mathrm{H}: \quad 2.2 \pm 0.9$ & $5.8 \pm 2.6$ & \multirow{2}{*}{9.2} & $39 \pm 20$ \\
\cline { 2 - 4 } & $\mathrm{F}: \quad 2$ & 4.8 & & \\
\hline \multirow{2}{*}{ (DNA)35ss } & $\mathrm{H}: \quad 1.5 \pm 0.2$ & $10.5 \pm 1$ & 12.5 & $57 \pm 5$ \\
\cline { 2 - 3 } & $\mathrm{F}: \quad 1.5$ & 10 & & \\
\hline
\end{tabular}

We can deduce that the (DNA) ${ }_{35 \mathrm{ss}}$ and (DNA) ${ }_{25 \mathrm{ss}}$ film lie down on the gold surface in air since the diameter of the strand can be assumed to $1 \mathrm{~nm}$. By contrast, a significant 
probability, $\mathrm{P}=20 \%$, is found to measure the inclination of the (DNA) ${ }_{15 \mathrm{ss}}$ of the order of $45^{\circ}$ towards the gold surface.

$\sigma_{\mathrm{DNA} 35 \mathrm{ss}}$ appears to be similar and even lower than $\sigma_{\mathrm{Au}}$. This suggests that the (DNA) ${ }_{35 s s}$ fully cover the gold surface, reducing the surface roughness by filling the grain boundaries. The flattening of the surface is confirmed by Figure 3A where the grain structure of gold is no longer visible.

Alternatively, $\sigma_{\text {DNA }}$ increases with decreasing the number of bases. In other words, the thicker the DNA film, the largest the standard deviation. This indicates that the shortest strands are more "mobile" than longer ones. Figure 3B is a typical AFM image representation of the (DNA) 15 ss immobilized on gold surface. The grain size of the gold structure is enlarged by $5 \mathrm{~nm}$ and some grains are nearly connected causing a smoothing of the image, although the columnar structure of gold is still easily recognizable contrary to the (DNA) 35 ss.

The standard deviation $\sigma_{\mathrm{Au}+\mathrm{DNA}}$ obtained from the Gaussian fitting of the histogram is indicative of the dispersion of the results corresponding to the thickness of both the gold and the DNA film. If we assume a linear relationship between the thickness of the gold and the DNA film, $\sigma_{\mathrm{DNA}}$ for the DNA film can be deduced in a first approximation from $\sigma_{\mathrm{Au}+\mathrm{DNA}}$ and $\sigma_{\mathrm{Au}}$ as follows:

$$
\sigma_{\mathrm{DNA}}^{2}+2 \sigma_{\mathrm{Au}} \sigma_{\mathrm{DNA}}=\sigma_{\mathrm{Au}+\mathrm{DNA}}^{2}-\sigma_{\mathrm{Au}}^{2}
$$

From Eq. 3 and values of Table $\mathbf{I}, \sigma_{\mathrm{DNA} 15 \mathrm{ss}}$ can be estimated to approximately $\sigma_{\mathrm{Au}+\mathrm{DNA} 15 \mathrm{ss}}-$ $\sigma_{\mathrm{Au}}=0.7 \mathrm{~nm}$. Since $95 \%$ of the samples in the Gaussian curve fall within $\pm 2 \sigma$ of the mean, it is convenient to assign a characteristic width of the distribution given by $4 \sigma$.

$\mu_{D N A}+2 . \sigma_{D N A}$ can be taken as physically signifying the maximum vertical distance from the gold surface. Note that for the $(\mathrm{DNA})_{15 \mathrm{ss}}, \mu_{\mathrm{DNA}}+2 . \sigma_{\mathrm{DNA}}=4.8 \mathrm{~nm}$ approaches the length of $5.8 \mathrm{~nm}$ expected for the unfold strands. 


\subsection{Forces in air between the DNA layer and the silicon nitride tip as a function of the}

sequence length.

The surface force profiles between the silicon nitride tip and the pads were measured. Raw data of the cantilever deflection corresponding to both the approach and the retraction of the tip from the DNA layer are shown in Figure 4A. The approach curve shown in the zoom-in of Figure $4 \mathrm{~A}$ is indicative of both the indentation depth and the mechanical properties of the biolayer.

The indentation depth is taken as the distance between the tip position corresponding to the maximum of the applied force and the contact point defined by the minimal force. The corresponding histograms obtained from 100 approach curves taken from different pads are shown in Figure 4B. The Gaussian distribution plotted for comparison does not fit the histogram over this entire range of depth, especially for the larger values on the right side of the curves. Note that the distribution of indentation depth for the (DNA) ${ }_{15 s s}$ is rather wide but matches with the expected values for unfold strands.

The mean values calculated from the Gaussian fit are comparable with values given in Table I but all are underestimated by $1 \mathrm{~nm}$. One possible error source might be attributed to the fact that we are not certain that the tip has reached the gold substrate. Measuring the pad thickness as shown in the previous section seems to be the best method to determine the DNA layer thickness.

An attempt to investigate the mechanical properties of the layer from the approach force profile was done using the Sneddon model. The effective Young's modulus, $E$, was calculated from the surface stiffness, $\mathrm{d} F / \mathrm{d} h$, and the penetration depth, $h$, as follows:

$$
E^{\prime}=\frac{1}{2} \sqrt{\frac{\pi}{a}} \frac{d F}{d h}
$$


where $A=4 \pi R h$ is the contact area of the tip and the surface (Stark et al., 1998) with $R$ the effective tip radius. The analysis of three typical approach curves is shown in Figure $\mathbf{4 C}$. First, E' is not constant over the full DNA film thickness. At the interface between gold and the DNA film, the gradient of the elastic modulus is rather sharp over $0.7 \mathrm{~nm}$ whatever the nature of the film. The mutual influence between the substrate and the film tends to increase the effective Young's modulus. Between 1 to $2 \mathrm{~nm}$ above the gold surface, E' appears to be constant, which means that in this range $E^{\prime}$ is not influenced by the substrate and the environment. The (DNA) ${ }_{15 s s}$ has a Young Modulus E' of approximately $100 \mathrm{MPa}$, lower than the two others strands. This can be justified by the higher stiffness of films composed by lying down strands since the layer is denser than in straight position. This is confirmed at the interface DNA/air, where the gradient of elastic modulus along the depth of the (DNA) $)_{15 s}$ expands vertically over $3 \mathrm{~nm}$, where the topmost surface is really soft with E' estimated to be below $10 \mathrm{MPa}$. This value can only be explained by a porous film which can be viewed as a composite defined from a DNA/air fraction.

The adhesion force at the contact between the silicon tip and the pads was measured from 100 retraction curves for each DNA film. The statistical distribution of adhesion forces, plotted in Figure 5A, clearly demonstrates that the Gaussian-fitted graph for native gold pads has a higher peak and a sharper distribution than DNA films. Increasing the number of bases results in a gradual decrease in the adhesion force. Since the adhesion event originates from van der Waals's forces, it can be thought of in terms of the Hamaker constant A. A film adsorbed on surface is known to reduce the overall Hamaker constant (Israelachvili, 1992).

In order to calculate the Hamaker constant between the gold substrate (index 2) and the silicon tip (index 1) separated by the DNA film (index 3), the following approximation can be expressed (Israelachvili, 1992): 


$$
A_{H}=A_{H 1}
$$

Hence, the Hamaker constants of the tip material (silicon) $A_{\mathrm{H} 131}$ and the gold material (metal) $A_{\mathrm{H} 232}$ have to be known. The Hamaker constant $A_{\mathrm{H} 131}$ between two bodies of a dielectric material 1 in a medium 3 can be calculated as follows (Israelachvili, 1992):

$$
A_{H 131}=\frac{3}{4}
$$

with $T$ being the temperature $(K), k$ the Boltzmann's constant $\left(k=1.381 \times 10^{-23} \mathrm{JK}^{-1}\right), h$ the Planck constant $\left(h=6.626 \times 10^{-34} \mathrm{Js}\right), \gamma$ the main absorption frequency in the UV range (a typical value is $\gamma \approx 3 \times 10^{15} \mathrm{~s}^{-1}$ ) and $n_{1}, n_{3}$ the optical index of the silicon tip and the DNA respectively. $A_{131}$ is mostly determined by its dispersion term (second term). Its entropic term (first term) is considered as negligible in assigning a reasonable dielectric constant between 4 and 20 to DNA strands. For metal particles, Eq. (6) does not hold because the dielectric constant is infinity. In this case, $A_{\mathrm{H} 232} \approx 4.10^{-19} \mathrm{~J}$ is a good approximation (Israelachvili, 1992).

If we assume that the contact between the parabolic shaped tip and the plane gold substrate is identical whatever the DNA film, the only difference between the adhesion forces originates from the changes in the optical index of the DNA film, $n_{3}$. It comes therefore that:

$$
\frac{F_{A u / D N A / S i}-F_{A u / A i r / S i}}{F_{A u / A i r / S i}}=\frac{A_{A u / D N A / S i}-A_{A u / A i r / S i}}{A_{A u / A i r / S i}}
$$

The optical index $n_{3}$ is then plotted as a function of the changes in adhesion forces (Figure $\mathbf{5 B}$ ). $n_{3}$ has been varied from 1 up to $n_{\mathrm{DNA}}=1.5$, which is a value commonly assigned to the crystalline form of the DNA. Figure 5B has been completed by the experimental measurements of the adhesion change. In this way, an experimental measurement of the optical index of the 
DNA film can be deduced. The high optical index of the (DNA) 35 ss, close to the value to the crystalline DNA, suggests a high density of this DNA film. The relationship between the surface coverage (DNA/air fraction) and the optical index can be roughly expressed as:

$$
n_{3}=n_{\text {air }}+\Gamma\left(n_{D N A}-n_{\text {air }}\right)
$$

where $\Gamma$ is the surface coverage.

The lowest change in adhesion forces for the (DNA) ${ }_{15 s s}$ is then readily explained by a surface coverage relatively low compared to the longer strands that adsorb in a side-on orientation. Moreover, changing the length of the DNA induces a corresponding proportional change in the area occupied per macromolecule.

\subsection{Forces in liquid between the DNA layer and the silicon nitride tip as a function of} the strand length.

Figure 6A shows the force profiles vs. the tip-sample separation measured in water on DNA film for three strand lengths. No force is acting on the probe at a separation larger than 15,17 and $25 \mathrm{~nm}$ from the surface for the (DNA) ${ }_{15 \mathrm{ss}},(\mathrm{DNA})_{25 \mathrm{ss}},(\mathrm{DNA})_{35 \mathrm{ss}}$, respectively.

Between these distances and the distance marked by an arrow on each curves, a repulsive force between the probe and the sample is present. An exponential behaviour clearly exists at long range distance regime. The long range regime can be explained by an electrostatic origin, due to the effective surface charge, which originates from water shielding. The approximate expression of the electrostatic force (called also double layer force) is given by (Butt et al., 1991):

$$
\mathrm{F}_{\text {elec }}=4 \pi . \operatorname{ch} . \mathrm{R} \psi_{\mathrm{T}} \exp \left(-K_{\mathrm{D}} \mathrm{D}\right)
$$

where $\mathrm{R}$ is the tip radius, ch is the surface charge, $\psi_{\mathrm{T}}$ is the tip potential and $K_{\mathrm{D}}$ is the inverse of the Debye length. $K_{\mathrm{D}}$ can be estimated to $0.1 \mathrm{~nm}^{-1}$. The long-range force does not seem to be affected by the strand length, as the three curves in Figure $\mathbf{6 A}$ are almost parallel and the 
magnitude of $\mathrm{F}_{\text {elec }}$ is similar. Since the tip radius is not well defined, it is difficult to estimate the strand density from the fit.

The biggest change in the force pattern is observed in the short-range regime, close to the contact with the DNA film. This second part of the curve attributed to the deformation of film shows a rather linear than exponential behaviour. The transition between the two regimes marked by arrows can be a good way to measure the thickness of the DNA film.

The histogram of the film thickness issued from the force analysis is shown in Figure 6B. The exponential to linear transition occurs at D $=5 \mathrm{~nm}, 6 \mathrm{~nm}$ and $13 \mathrm{~nm}$ for (DNA) $15 \mathrm{ss}$, (DNA) ${ }_{25 \mathrm{ss}}$ $(\text { DNA })_{35 s s}$ respectively (Figure 6A). Note that the thickness has an inverse order with what was measured in air. The increase in the film thickness in liquid whatever the strand length is an evidence of structural rearrangements: strands straighten up i.e. inclination angle decreases.

The repulsive force at the contact of the sample is $1 \mathrm{nN}$, a value that is comparable with what was measured for the indentation in air. The repulsive force in contact with gold is however higher for longer strands. In addition, the force profile appears different especially for (DNA) ${ }_{35 \mathrm{ss}}$, where different interaction can be discerned according the indentation range: one at shorter range between $0.5 \mathrm{~nm}$ and $5 \mathrm{~nm}$ and another between $5 \mathrm{~nm}$ and $12 \mathrm{~nm}$. We attribute the first regime to the compression of the film as classically treated by common elastic mechanical models, such as Hertz's theory or any other models based on continuum mechanics, but also to nanoscale structural changes in the DNA film. We believe the second regime corresponds to the steric force (Meagher et al., 2002). A molecular approach is thus more appropriate to treat the longer range of the indentation. 


\subsection{Thickness distribution of the DNA film in liquid}

It is interesting to compare the force analysis and the height measured by image crosssection. In AFM, we always try to minimize the applied force as lower as possible (approximately $10 \mathrm{pN}$ in liquid). The interpretation of the layer thickness in liquid is more complex since repulsive electrostatic forces can be detected above the layer and therefore the thickness can be overestimated. Nevertheless, the height difference observed in AFM can be used to gain information on the change in layer thickness if the applied force remains unchanged during the entire scan time.

The applied force was maintained at the transition regime defined in the last section (marked by an arrow in Figure 6A).

Figure 2B shows the height distribution measured in liquid. Results are reported in Table I.

Nanoindentation depth for a typical force of $1 \mathrm{nN}$ is consistent with the thickness of the DNA layer (Table II) obtained from AFM image cross-section. A difference in coherence appears to be more marked for shorter strands, certainly due to the greater mobility of these shorter strands. The strands mobility can be compared using the term $2 . \sigma_{\mathrm{DNA}}$, which corresponds to the incertitude for the DNA thickness in Table II. In liquid, 2. $\sigma_{\mathrm{DNA}}(15 \mathrm{ss})$ has roughly the same order of magnitude than the mean value $\mu$ DNA(15ss) whereas $2 . \sigma_{\mathrm{DNA}(35 \mathrm{ss})}$ represents only $10 \%$ of $\mu_{\mathrm{DNA}(15 \mathrm{ss})}$; the film of longer strands appears more structured than those of shorter strands.

The effective orientation $\alpha$ of the strands towards the substrate plane can be estimated by the ratio between $\mu_{\mathrm{DNA}}$ and the expected length of strands $L$. The mean value $\alpha$ appears to increase with the number of bases, but the standard error is more important for shortest strands. The sum of the standard error and of the mean value remains constant to approximately $60^{\circ}$ whatever the number of bases. 
Comparative analysis between the orientations of the strands in air and in liquid indicates that the mobility of the strands is governed by electrostatic interactions. The effective DNA charge density is considerable: about one negative charge per base namely roughly $2 \mathrm{e}^{-}$ $/ \mathrm{nm}^{3}$ whereas the gold substrate is positively charged. The fact that longer strands lie down on the gold surface in air and stand up in liquid indicates that the electrostatic attraction is considerably reduced in aqueous solution compared to air. Most of the electrostatic attraction becomes negligible in water by screening the charge density of gold substrate. The fact that the (DNA) ${ }_{35 \mathrm{ss}}$ has the highest change in $\mu_{\mathrm{DNA}}$, coupled with the lowest standard error 2. $\sigma_{\mathrm{DNA}}$, indicates that the longer strands are maybe repulsed by the gold surface, which imposes a charge reversal of the gold surface in liquid by ionic adsorption and a decrease in the (DNA) ${ }_{35 \text { ss }}$ mobility.

\section{Conclusion}

The Atomic Force Microscopy was systematically used to investigate the immobilization of the DNA single strands onto gold both in air and in liquid. Analysis of gold pads patterned onto a silicon surface was found to be a reliable method to measure the DNA thickness distribution (mean value and standard error).

Interestingly, our results also suggest that the height of the DNA strand depends on both the number of bases and the environment. In air, longer strands, whose surface electrostatic charge is the highest lay down onto the gold surface, positively charged surface. In liquid, the charge reversal of gold causes a repulsion of longer strands that stand up.

Those measurements were compared to results obtained by AFM but in its force measurement mode. The change in adhesion forces in air attributed to the change in Van der Waals forces 
was used to locally examine the surface coverage of the film. Longer strands appear to cover the entire surface. The nanoindentation of the film is consistent to cross-section analysis of pads. Analysis of the force measurement in liquid (compression, steric and electrostatic) was found instructive to analyse the AFM image.

\section{Acknowledgments}

This work was supported by the CNRS program dealing "Protéomique et génie des protéines". 


\section{Caption}

Fig. 1: Characterization of the gold pads before DNA immobilization. (a) Typical AFM image obtained on native gold surface. (b) AFM image of the $4 \times 4$ array of gold pads deposited on silicon substrate. (c) Typical histogram of the pad thickness with a bin size of $0.1 \mathrm{~nm}$. The mean value of the gold pad thickness is measured between 54.6 and $54.9 \mathrm{~nm}$ with a standard deviation $\sigma$ of $1.2 \mathrm{~nm}$. (d) Standard deviation $\sigma$ plotted as a function of the gold surface area analysed. Dots represent the measurement performed with varying the number of pads. The solid line is given by the surface roughness (RMS) obtained from a continuous film.

Fig. 2: Height distributions for the 15, 25 and 35 strands grafted on gold pads in air (a), in liquid (b).

Fig. 3: AFM image of DNA strands immobilized on gold surface. (b) (DNA) 35 ss fully covers the gold surface, reducing the surface roughness by filling the grain boundaries. The surface is flattened and the grain structures of gold is no longer visible. (c) Concerning (DNA) ${ }_{15 s s,}$ the grain size of the gold structure is enlarged by $5 \mathrm{~nm}$ and some grains are almost connected causing a smoothing of the image, but the columnar structure of gold is still easily recognizable contrary to the $(\mathrm{DNA})_{35 \mathrm{ss}}$.

Fig. 4: (a) Typical surface force profiles in air between the silicon nitride tip and the gold pads. Cantilever deflection corresponding to both the approach and the retraction of the tip are plotted vs. the piezoelectric displacement. The force F between the tip and the pad plotted as a function of the distance $\delta \mathrm{D}$ separating the pad and the tip was obtained from the zoom-in of the approach curve. (b) Distribution of the indentation depth of pads grafted by (DNA) $35 \mathrm{ss,}$ $(\text { DNA })_{25 s,}$, DNA $)_{15 s s}$ in air issued from 100 force analyses on each pad. Gaussian distribution is plotted on solid line for comparison. (c) Evolution of the Young modulus E' calculated using Eq. 4 from the force vs. separation profile (Fig. 6) as a function of the penetration depth 
in the three DNA films in air. The (DNA) $15 \mathrm{ss}$ has a Young Modulus E' $(\approx 100 \mathrm{MPa})$ lower than the two others strands.

Fig. 5: Analyses of the adhesion forces in air (a) Statistical distribution of adhesion forces at the contact between the silicon tip and the pads. The Gaussian-fitted graph for native gold pads has a higher peak and sharper distribution than DNA films. Increasing the number of bases results in a gradual decrease in the adhesion force. (b) The solid line represents how the change in adhesion force varies with the optical index of the layer following the Eq. 5,6 and 7. The surface coverage is obtained from the change in optical index by means of Eq. 8 . Experimental data are shown using dot lines

Fig. 6: (a) Typical surface force profiles measured in water on DNA film for three strand lengths. The transition between the two regimes, marked by arrows, can be a good way to measure the thickness of the DNA film. (b) Distribution of the indentation depth of pads grafted by (DNA) 35 ss, $\left(\right.$ DNA) $25 \mathrm{ss}$, (DNA) ${ }_{15 \mathrm{ss}}$ in liquid issued from 100 force analyses on each pad. Gaussian distribution is plotted on solid line for comparison. 

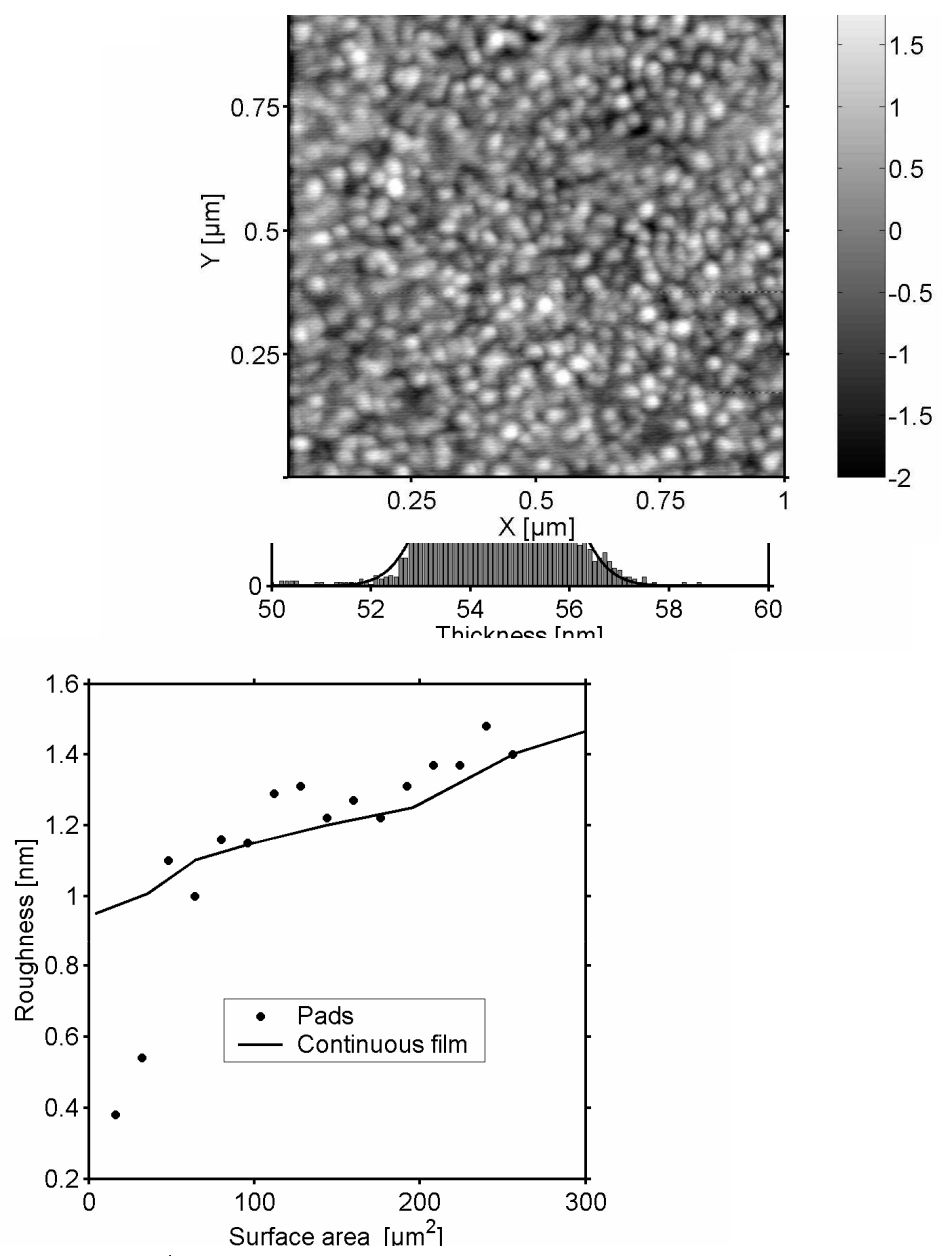

A

C

FIGURE 1

G. LEGAY \& al.

Biosensors \& Bioelectronics

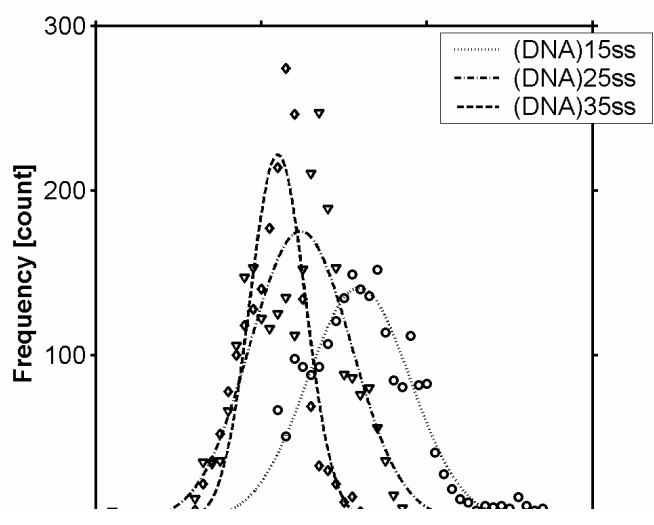


A

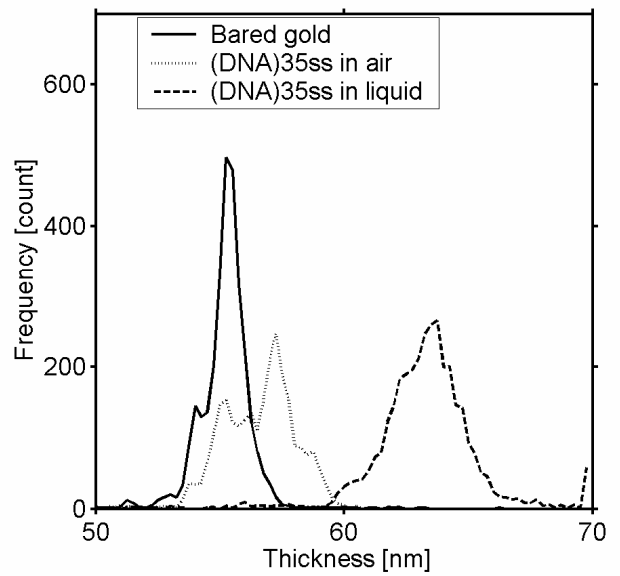

FIGURE 2

G. LEGAY \& al.

Biosensors \& Bioelectronics

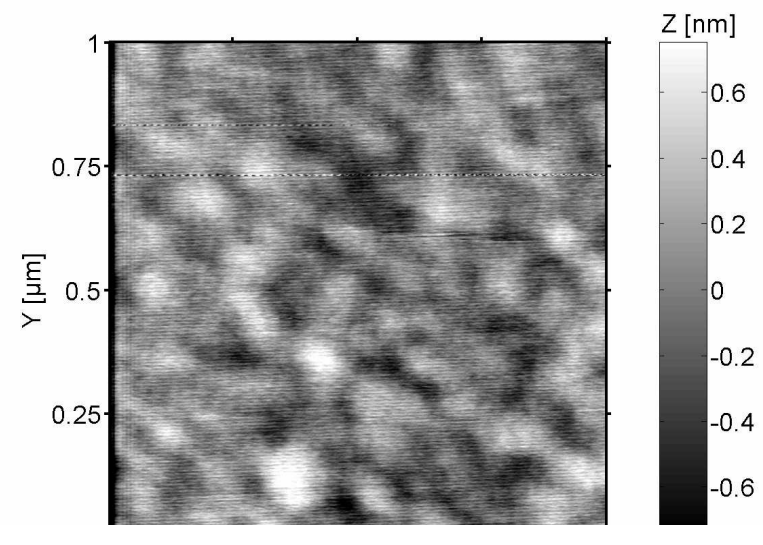




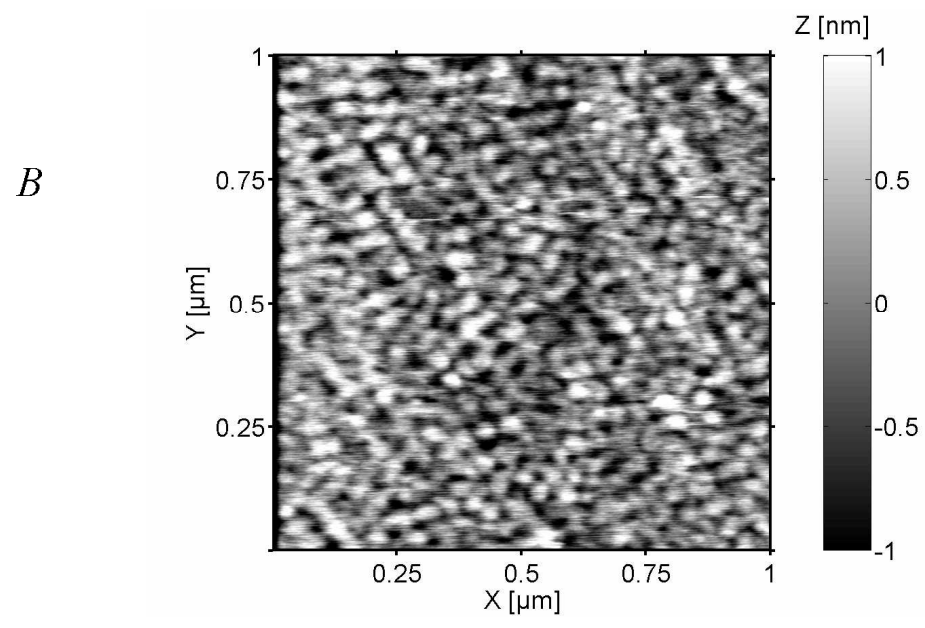

FIGURE 3

G. LEGAY \& al.

Biosensors \& Bioelectronics

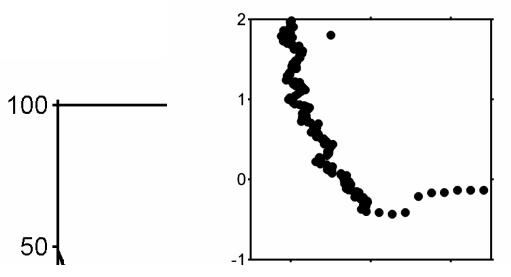



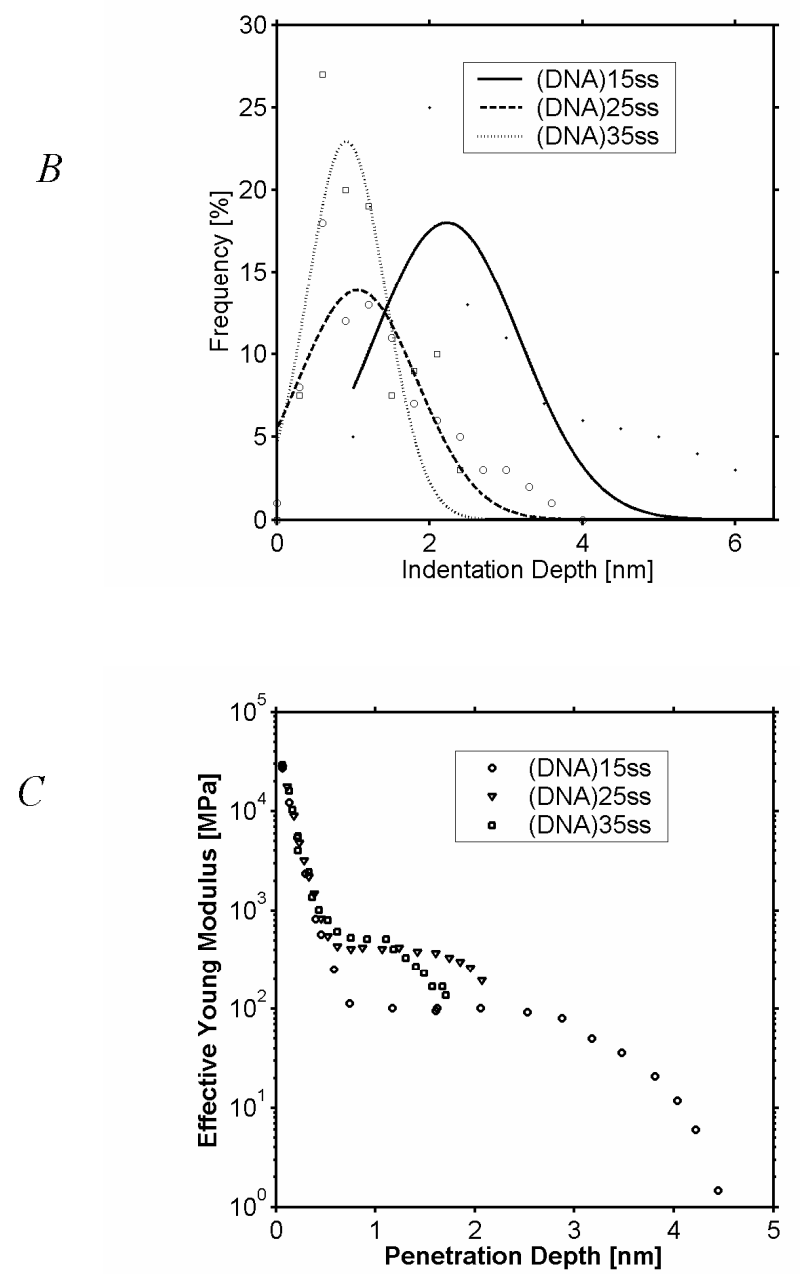

FIGURE 4

G. LEGAY \& al. Biosensors \&

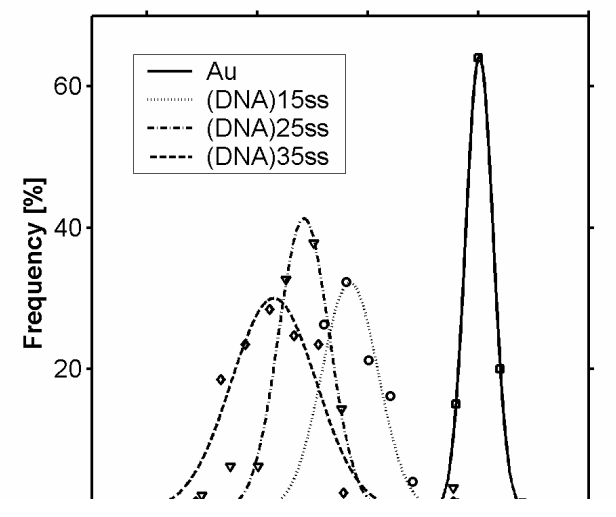

Bioelectronics 
$B$

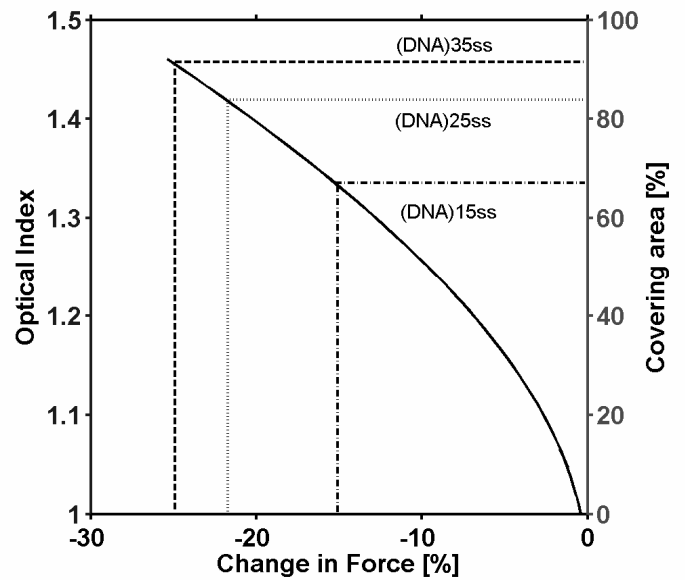

FIGURE 5

G. LEGAY \& al.

Biosensors \& Bioelectronics 
A

$B$

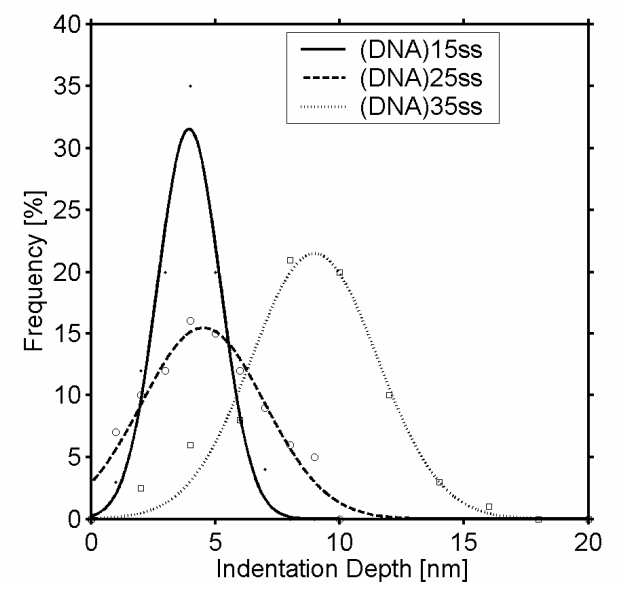

FIGURE 6

G. LEGAY \& al.

Biosensors \& Bioelectronics 


\section{References}

Bassi1, N., Maillart, E., Canva, M., Lévy, Y., Millot, M., Pissard, S., Narwa, R., Goossens, M., 2003. One hundred spots parallel monitoring of DNA interactions by SPR imaging of polymer-functionalized surfaces applied to the detection of cystic fibrosis mutations. Sensor and Actuators B 94(3), 313-323

Butt, H.J., 1991. Measuring electrostatic, van der Waals, and hydration forces in electrolyte solutions with an atomic force microscope. Biophys. J. 60, 1438-1444.

Carpini, G., Lucarelli, F., Marrazza, G., Mascini, M., 2004. Oligonucleotide-modified screenprinted gold electrodes for enzyme-amplified sensing of nucleic acids. Biosensors and Bioelectronics 20(2), 167-175

Cherian, S., Gupta, R.K., Mullin, B.C., Thundat, T., 2003. Detection of heavy metal ions using protein-functionalized microcantilever sensors. Biosensors and Bioelectronics 19(5), 411-416.

Cho, Y.K., Kim, S., Lim, G., Granick, S., 2001. A surface forces study of DNA hybridization. Langmuir 17, 7732-7734.

Finot, E., Bourillot, E., Meunier-Prest, R., Lacroute, Y., Legay, G., Cherkaoui-Malki, M., Latruffe, N., Siri, O., Braunstein, P., Dereux, A., 2003. Performance of interdigitated nanoelectrodes for electrochemical DNA biosensor. Ultramicroscopy 97(1-4), 441-449.

Georgiadis, G., Peterlinz, K.P., Peterson, A.W., 2000. Quantitative measurements and modeling of kinetics in nucleic acid monolayer films using SPR spectroscopy. Am. Chem. Soc. 122(13) 3166-3173.

Gray, D.E., Case-Green, S.C., Fell, T.S., Dobson, P.J. \& Southern, E.M., 1997. Ellipsometric and interferometric characterization of DNA probes immobilised on a combinatorial array. Langmuir 13, 2833-2842.

Holmberg, M., Kühle, A., Garneas, J., Boisen, A., 2003. Hybridisation of short DNA molecules investigated with in situ atomic force microscopy. Ultramicroscopy 97(1-4), $257-$ 261.

Israelachvili, J., 1992. Intermolecular and Surface Forces, Academic Press, London.

Lenigk, R., Carles, M., Ip, N.Y., Sucher, N.J., 2001. Surface characterization of a siliconchip-based DNA microarray. Langmuir 17(8), 2497-2501.

Levicky, R., Herne, T.M, Tarlov, M.J., Satija, S.K., 1998. Using Self-Assembly to Control the Structure of DNA Monolayers on Gold: A Neutron Reflectiviity Study. J. Am. Chem. Soc. $120,9787-9792$. 
Liang, Z., Xie, W., Zhang, X., Chen, J., Zhang, X., Danielsson, B., Mecklenburg, M., Xie, B., 2004. Electrochemical study of the XNA on Gold ${ }^{\mathrm{TM}}$ microarray. Biosensors and Bioelectronics 20(2) 211-216.

Lotierzo, M., Henry, O.Y.F., Piletsky, S., Tothill, I., Cullen, D., Kania, M., Hock, B., Turner, A.P.F., 2004. Surface plasmon resonance sensor for domoic acid based on grafted imprinted polymer. Biosensors and Bioelectronics 20(2), 145-152.

Marie, R., Jensenius, H., Thaysen, J., Christensen, C.B., Boisen, A., 2002. Detection of heavy metal ions using protein-functionalized microcantilever sensors. Ultramicroscopy 91(1-4), 2936.

Meagher, L., Griesser, H.J., 2002. Interactions between adsorbed lactoferrin layers measured directly with the atomic force microscope. Colloids and Surfaces B: Biointerfaces 23(2-3), 125-140.

Meunier-Prest, R., Raveau, S., Finot, E., Legay, G., Cherkaoui-Malki, M., Latruffe, N., 2003. Direct measurement of the melting temperature of supported DNA by electrochemical method. Nucleic Acids Research 31(23).

Moreno-Herrero, F., Colchero, J., Baro, A.M., 2003. DNA height in scanning force microscopy. Ultramicroscopy 96(2), 167-174.

O'Brien, J.C., Stickney, J.T., Porter, M.D., 2000. Preparation and Characterization of SelfAssembled Double-Stranded DNA (dsDNA) Microarrays for Protein:dsDNA Screening Using Atomic Force Microscopy. Langmuir 16(24), 9559-9567.

Rasmussen, P.A, Thaysen, J., Hansen, O., Eriksen, S.C., Boisen, A., 2003. Optimised cantilever biosensor with piezoresistive read-out. Ultramicroscopy 97(1-4), 371-376.

Satjapipat, M., Sanedrin, R., Zhou, F., 2001. Selective Desorption of Alkanethiols in Mixed Self-Assembled Monolayers for Subsequent Oligonucleotide Attachment and DNA Hybridization. Langmuir 17(24),7637-7644.

Schotanus, M.P., Aumann, K.S., Sinniah, K., 2002. Using Force Spectroscopy To Investigate the Binding of Complementary DNA in the Presence of Intercalating Agents. Langmuir 18(14), 5333-5336.

Shi, X., Sanedrin, R.J., Zhou, F., 2002. Structural Characterization of Multilayered DNA and Polylysine Composite Films: Influence of Ionic Strength of DNA Solutions on the Extent of DNA Incorporation. J. Phys. Chem. B 106(6), 1173-1180.

Stark, R.W., Drobek, T.,Weth, M., Frickef, J., Heckel, W.M., 1998. Determination of elastic properties of single aerogel powder particles with the AFM. Ultramicroscopy 75(3), 161-169.

Ulman, A., 1991. An Introduction to Ultrathin Organic Films from Langmuir-Blodgett to Self-Assembly. Harcourt Brace Janovitch, Boston. 
Ulman, A., Kang, J.F., Shnidman, Y., Liao, S., Jordan, R., Choi, G.Y., Zaccaro, J., Myerson, A.S., Rafailovich, M., Sokolov, J., Fleischer, C., 2000, Self-assembled monolayers of rigid thiols. Reviews in Molecular Biotechnology 74(3), 175-188.

Wang, J., Bard, A.J., 2001. Monitoring DNA Immobilization and Hybridization on Surfaces by Atomic Force Microscopy Force Measurements. Analyitical Chemistry 73, 2207-2212.

Yoshino, T., Sugiyama, S., Hagiwara, S., Fukushi, D., Shichiri, M., Nakao, H., Kim, J.M., Hirose, T., Murumatsu, H., Ohtani, T., 2003. Nano-scale imaging of chromosomes and DNA by scanning near-field optical/atomic force microscopy. Ultramicroscopy 97(1-4), 81-87.

Zhou, D., Sinniah, K., Abell, C., Rayment, T., 2002. Use of Atomic Force Microscopy for Making Addresses in DNA Coatings. Langmuir 18(22), 8278-8281. 